\title{
Nutritional status and physical activity level predict Timed Up and Go performance in Irish older adults
}

\author{
M.L. O'Connell ${ }^{1}$, T. Coppinger ${ }^{2}$, J. Walton ${ }^{1}$ and A.L. McCarthy ${ }^{1}$ \\ ${ }^{1}$ Department of Biological Sciences, Cork Institute of Technology, Rossa Avenue, Bishopstown, Cork and \\ ${ }^{2}$ Department of Sport, Leisure and Childhood Studies, Cork Institute of Technology, Rossa Avenue, Bishopstown, Cork, \\ Ireland
}

Functional decline is a common feature of ageing, and is associated with a tripled risk of mortality, higher healthcare expenses and reduced quality of life ${ }^{(1)}$. Good nutrition and regular physical activity (PA) may help to modulate this physiological deterioration and preserve independence in older adults, adding quality to life years. The Timed Up and Go Test (TUG) is a measure of functional ability that correlates to mobility and falls risk ${ }^{(2)}$. The aim of this study was to enhance our understanding of contributing factors to functional decline by investigating the relationship between PA, nutritional status and TUG performance in a cohort of Irish adults aged $\geq 65$ years.

This cross-sectional study included 162 community-dwelling older adults $(n=92$ female, $n=70$ male, $73.8 \pm 6.66$ years $)$ who responded to advertisements and information sessions delivered in community centres in Cork, Southern Ireland. Health screening sessions took place in community settings across Cork. TUG (seconds) was measured using standard protocol ${ }^{(2)}$. A TUG time of $>10$ seconds was used to detect impaired mobility ${ }^{(3)}$. Nutritional status was assessed using the Mini Nutritional Assessment Short Form (MNA-SF). PA level was measured using the Physical Activity Scale for the Elderly (PASE), with minor adaptations to ensure suitability for an Irish population. The relationship between nutritional status, PA level and TUG time was analysed using multiple linear regression analyses adjusted for age (years), body mass index (BMI, $\mathrm{kg} / \mathrm{m} 2$ ), area of residence (urban/rural), gender and alcohol intake (g/day). Ethical approval was obtained from Cork Institute of Technology Research Ethics Committee.

The average TUG time was $9.05 \pm 1.98$ s for males and $9.88 \pm 2.69$ s for females. Overall, $30.9 \%$ of participants had impaired mobility (TUG $>10 \mathrm{~s}$ ). A total of $1.2 \%$ and $19.1 \%$ of the study population were malnourished and at risk of malnutrition, respectively. Males were more physically active than females $(\mathrm{p}=0.023)$. Almost half of the variation in TUG was explained by the predictors included in the linear regression model $(\mathrm{R} 2=49.1 \%)$. Increasing age $(\beta=0.496 ; p<0.001)$ and higher $\mathrm{BMI}(\beta=0.166 ; \mathrm{p}=0.004)$ were positively associated with TUG, while male gender $(\beta=-0.124 ; p=0.039)$, urban residence $(\beta=-0.146$; $p=0.015)$, PASE score $(\beta=-0.231 ; p<0.001)$ and MNA-SF score $(\beta=-0.171 ; p=0.003)$ were inversely associated with TUG.

The results of this study suggest that poorer nutritional status and lower PA level are significant predictors of physical function as measured by TUG in this cohort; in addition to increasing age, female gender, higher BMI and rural residence. Addressing nutritional needs and promoting PA are important considerations for functional preservation strategies in Irish older adults.

\section{Acknowledgments}

We would like to thank Cork Institute of Technology for providing the funding for this research, and all of the participants of this study for their time.

\section{References}

1. Hébert R (1997) Can Med Assoc J, 157, 1037-45.

2. Shirley Ryan Ability Lab (2013) Rehabilitation Measure Database: Timed Up and Go

3. Lee JE, Chun H, Kim YS et al. (2020) J Korean Med Sci, 35, e25. 